\title{
Exact Mean-Field Theory Explains the Dual Role of Electrical Synapses in Collective Synchronization
}

\author{
Ernest Montbrió $\odot^{1}$ and Diego Pazó $\odot^{2}$ \\ ${ }^{1}$ Department of Information and Communication Technologies, Universitat Pompeu Fabra, 08003 Barcelona, Spain \\ ${ }^{2}$ Instituto de Física de Cantabria (IFCA), CSIC-Universidad de Cantabria, 39005 Santander, Spain
}

(Received 1 September 2020; revised 18 November 2020; accepted 18 November 2020; published 10 December 2020)

\begin{abstract}
Electrical synapses play a major role in setting up neuronal synchronization, but the precise mechanisms whereby these synapses contribute to synchrony are subtle and remain elusive. To investigate these mechanisms mean-field theories for quadratic integrate-and-fire neurons with electrical synapses have been recently put forward. Still, the validity of these theories is controversial since they assume that the neurons produce unrealistic, symmetric spikes, ignoring the well-known impact of spike shape on synchronization. Here, we show that the assumption of symmetric spikes can be relaxed in such theories. The resulting mean-field equations reveal a dual role of electrical synapses: First, they equalize membrane potentials favoring the emergence of synchrony. Second, electrical synapses act as "virtual chemical synapses," which can be either excitatory or inhibitory depending upon the spike shape. Our results offer a precise mathematical explanation of the intricate effect of electrical synapses in collective synchronization. This reconciles previous theoretical and numerical works, and confirms the suitability of recent low-dimensional mean-field theories to investigate electrically coupled neuronal networks.
\end{abstract}

DOI: 10.1103/PhysRevLett.125.248101

Electrical coupling via gap junctions is broadly present across brain areas [1-5]. There is ample experimental evidence that gap junctions are involved in synchronizing inhibitory networks [6-15]. Yet, despite the fact that electrical synapses are recognized to constitute a critical component of the brain, the function of these junctions and the mechanisms whereby they contribute to synchrony are subtle and not well understood $[4,15]$.

Unlike chemical synapses, the transmission of action potentials via gap junctions greatly depends upon the overall shape of the action potential. This makes the effects of electrical coupling far from trivial, and the theoretical and computational analysis of electrically coupled networks of spiking neurons a difficult task. Previous theoretical studies identified both the shape of the spikes and the firing frequency of the neurons as key parameters influencing synchrony [16-22]. Yet, a precise mechanistic explanation of the role of these parameters in synchrony is lagging, and different works often provide results that are difficult to reconcile. For example, studies on homogeneous, twoneuron networks invoke the weak coupling limit and find that electrical and chemical synapses combine their effect in a linear manner [17,18]. Moreover, the shape of the spikes determines whether electrical synapses cooperate or compete with inhibition for synchrony [18]. In contrast, works investigating large heterogeneous networks suggest that electrical and inhibitory synapses play distinct roles: While strong electrical coupling leads to collective synchrony [22-24], strong inhibition typically leads to the suppression of firing and destroys synchrony [25-28].
Rather than investigating the dynamics of large populations of spiking neurons, an alternative and widespread theoretical approach is to use so-called mean-field models (also called firing-rate or neural-mass models) [29]. Such models are simplified, low-dimensional mathematical descriptions of the mean activity of the population but they only characterize populations with chemical synapses. Yet, an important theoretical achievement linking individual and global dynamics has been recently accomplished with the advent of the Ott-Antonsen theory [30], and with its application to populations of $\theta$ neurons [31] and quadratic integrate-and-fire (QIF) neurons [32]. Notably, these novel theoretical approaches readily apply to networks with electrical synapses [33-36].

Unfortunately, the mean-field theories proposed in [31,32] rely on an assumption of critical importance for electrically coupled networks: Spikes need to have a very particular symmetric shape that is unrealistic [see Fig. 1(a)], and this may largely alter the network dynamics. As we show below this is the reason why the low-dimensional firing-rate equations (FREs) for electrically coupled networks originally derived in [33,34] fail to elucidate how chemical and electrical couplings might add their effects linearly, as found in $[17,18]$. Therefore, though the meanfield theories in $[31,32]$ have been successfully applied to investigate networks with chemical synapses [28,37-57], their suitability to investigate synchrony in networks with gap junctions remains questionable.

In this Letter, we show that the mean-field theory originally proposed in [32] can be generalized to account 

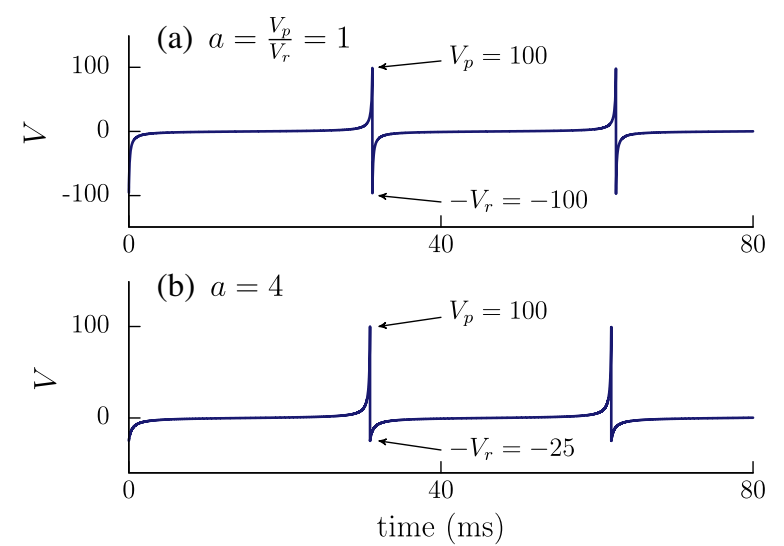

FIG. 1. Time series of an oscillatory QIF neuron, Eq. (1), with (a) symmetric, and (b) nonsymmetric resetting rule. $\eta=1$, $\tau=10 \mathrm{~ms}$.

for nonsymmetric spikes. Remarkably, this leads to an extension of the FREs in [34], which provides the first precise mechanistic explanation of the dual role of electrical synapses in collective synchrony. This explanation reconciles previous disparate theoretical and numerical results on the intricate effects of electrical coupling in synchrony, and consolidates the FREs for QIF neurons as a valid and singular low-dimensional description for populations of electrically coupled neurons.

Quadratic integrate-and-fire neuron.-First of all, we introduce the QIF model, and explain the main idea to generalize the mean-field theory developed in [32]. The model describes the evolution of the membrane potential variable $V$ via the first-order ordinary differential equation [58-60]

$$
\tau \dot{V}=V^{2}+\eta ; \quad \text { if } V>V_{p} \quad \text { then } V \leftarrow-V_{r} .
$$

The overdot denotes the derivative with respect to time, $\tau$ is the membrane's time constant, and $\eta$ represents an external current. Because of the quadratic nonlinearity in Eq. (1), $V$ may escape to infinity in a finite time. To prevent this, the QIF model incorporates a resetting rule: Each time the neuron's membrane potential $V$ reaches the peak value $V_{p}$, the neuron emits a spike and the voltage is reset to $-V_{r}$ (here we consider $V_{p}, V_{r}>0$ ), see Fig. 1.

It is convenient to define a positive, real parameter characterizing spike asymmetry as the ratio [61]

$$
a \equiv \frac{V_{p}}{V_{r}} .
$$

When $a=1$, the resetting rule of the QIF model is symmetric, see Fig. 1(a). This symmetry is implicitly assumed in the transformation between the QIF and the $\theta$-neuron models, which can be formally performed in the limit $V_{r}=V_{p} \rightarrow \infty$ [62].
The mean-field theory in [32] assumes symmetric resetting, $a=1$, and then adopts the limit $V_{r} \rightarrow \infty$. We next show that the assumption of symmetric resetting can be relaxed in this theory, leading to a novel low-dimensional firing-rate model with arbitrary spike asymmetry.

Population model.-We investigate a population of QIF neurons

$$
\tau \dot{V}_{j}=V_{j}^{2}+\eta_{j}+J \tau r(t)+g\left[v(t)-V_{j}\right],
$$

with the resetting rule of Eq. (1). The index $j=1, \ldots, N$ labels all the neurons in the population, and the parameters $\eta_{j}$ represent external currents that are taken from some prescribed probability distribution $G(\eta)$.

In Eq. (3) electrical coupling, of strength $g \geq 0$, diffusively couples each neuron's membrane potential with the mean membrane potential $v(t)=(1 / N) \sum_{j=1}^{N} V_{j}(t)$. Additionally, each neuron in the population is connected to all the other neurons via chemical synapses of strength $J$. Electrical synapses typically connect inhibitory neurons and hence $J$ should be thought of as a negative parameter. Chemical coupling is mediated by the average firing rate $r(t)=1 /\left(N \tau_{s}\right) \sum_{j=1}^{N} \sum_{k} \int_{t-\tau_{s}}^{t} d t^{\prime} \delta\left(t^{\prime}-t_{j}^{k}\right)$, where $t_{j}^{k}$ is the time of the $k$ th spike of the $j$ th neuron, $\delta(t)$ is the Dirac delta function, and $\tau_{s} \ll 1$.

Theoretical analysis.-Next, we perform the thermodynamic limit, drop the indices of Eqs. (3), and define the time-dependent conditional density $\rho(V \mid \eta, t)$, such that $\rho(V \mid \eta, t) d V$ is the fraction of neurons with membrane voltage between $V$ and $V+d V$, and heterogeneity parameter $\eta$ at time $t$. The density $\rho$ necessarily obeys the continuity equation

$$
\tau \partial_{t} \rho+\partial_{V}\left\{\left[V^{2}+\eta+J \tau r+g(v-V)\right] \rho\right\}=0 .
$$

This equation can be solved resorting to the Lorentzian ansatz (related to the Ott-Antonsen ansatz [30] through a conformal mapping [32])

$$
\rho(V \mid \eta, t)=\frac{1}{\pi} \frac{x(\eta, t)}{[V-y(\eta, t)]^{2}+x(\eta, t)^{2}},
$$

which is the asymptotic shape of the density in the long time limit assuming that $V$ spans over the whole real line $[32,34]$. We note that in numerical simulations $V \in\left(-V_{r}, V_{p}\right)$, and therefore the Lorentzian ansatz Eq. (5) is an approximation that works progressively better as $V_{p}, V_{r} \rightarrow \infty$.

Inserting Eq. (5) into Eq. (4), we get the evolution equations for $x(\eta, t)$ and $y(\eta, t)$. Nevertheless, before doing so, it is convenient to express the global quantities $r(t)$ and $v(t)$ in Eq. (4) in terms of $x(\eta, t)$ and $y(\eta, t)$. Regarding the mean firing rate $r$, it is related with the width $x(\eta, t)$ of the Lorentzian ansatz [32]. Indeed, the firing rate for neurons with a given $\eta$ value is the probability flux at $V_{p}$ (taken at infinity), which gives the identity 
$r(\eta, t)=x(\eta, t) /(\pi \tau)$. Then, the mean firing rate $r(t)$ is given by the integral over all $\eta$

$$
r(t)=\frac{1}{\pi \tau} \int_{-\infty}^{\infty} x(\eta, t) G(\eta) d \eta
$$

Mean membrane potential for general resetting rule.Next, we show that, for general resetting, the mean membrane potential $v$ depends on the central voltages $y(\eta, t)$ as well as on the widths $x(\eta, t)$ in Eq. (5). This combined dependence underlies the dual effects of electrical synapses.

The average voltage for neurons with a certain $\eta$ value can be calculated taking the following limit [63]:

$$
v(\eta, t)=\lim _{V_{r} \rightarrow \infty} \int_{-V_{r}}^{V_{p}=a V_{r}} \rho(V \mid \eta, t) V d V .
$$

The mean-field theory originally proposed in [32] considers $a=1$. However, here we relax this assumption and split the integral in Eq. (7) into two parts: one integral with symmetric integration limits (Cauchy principal value), and another one with the remaining integration interval

$$
v(\eta, t)=\left[\text { p.v. } \int_{-\infty}^{\infty}+\lim _{V_{r} \rightarrow \infty} \int_{V_{r}}^{a V_{r}}\right] \rho(V \mid \eta, t) V d V .
$$

The first integral simply yields the center of the distribution of membrane potentials $y(\eta, t)$, see Eq. (5). The second integral is the contribution to the mean membrane voltage due to asymmetric spike resetting, and it can be evaluated in closed form. Then, after taking the limit, we find

$$
v(\eta, t)=y(\eta, t)+\frac{\ln a}{\pi} x(\eta, t) .
$$

This identity pinpoints the deviation of the mean membrane potential $v(\eta, t)$, with respect to the center of the distribution of voltages $y(\eta, t)$, due to asymmetric spike resetting, $a \neq 1$. Remarkably, this deviation is both proportional to the firing rate through $x(\eta, t)$, and to $\ln a$. Therefore, for spikes with $a>1$, the mean membrane voltage is above the center of the distribution, whereas for spikes with $0<a<1$ the mean membrane voltage is below $y(\eta, t)$.

For symmetric resetting, the mean membrane potential $v_{s}(t)$ of the entire population is obtained integrating $y(\eta, t)$ over all $\eta$ values:

$$
v_{s}(t) \equiv \int_{-\infty}^{\infty} y(\eta, t) G(\eta) d \eta .
$$

In the general case of asymmetric spike resetting, the mean membrane voltage is obtained integrating Eq. (9) over $\eta$. Hence, using Eqs. (6) and (10), we find

$$
v(t)=v_{s}(t)+(\tau \ln a) r(t) .
$$

Dynamics in the "Lorentzian manifold".-The evolution equations for $x(\eta, t)$ and $y(\eta, t)$, obtained substituting Eq. (5) into Eq. (4), can be condensed into one via the complex variable $w(\eta, t) \equiv x(\eta, t)+i y(\eta, t)$ :

$$
\tau \partial_{t} w(\eta, t)=i\left[\eta+(J+g \ln a) \tau r-w^{2}\right]+g\left(i v_{s}-w\right),
$$

where $r$ and $v_{s}$ are the integrals in Eqs. (6) and (10). Note that Eq. (12) is an infinite dimensional system, since each $w(\eta, t)$ is coupled to all the other functions $w\left(\eta^{\prime}, t\right)$.

Three results follow from Eq. (12), which are valid irrespective of the specific form of the distribution $G(\eta)$. First, asymmetric spike resetting influences the collective dynamics of the network only in the presence of electrical coupling $g \neq 0$. Second, the term $\ln a$ weights the "virtual chemical coupling," which linearly adds to the actual chemical coupling constant $J$. Moreover, the sign of $\ln a$ determines if this contribution is excitatory or inhibitory. Thus, in the presence of electrical coupling, the effective chemical coupling constant becomes

$$
J_{\text {eff }}(J, g, a)=J+g \ln a .
$$

Third, terms with electrical coupling $g$ appear at two different places in Eq. (12), indicating a dual role of gap junctions in the dynamics of $w$.

Firing-rate equations. - To gain further insight into the effects of electrical coupling we adopt hereafter Lorentzian distributed currents with center at $\bar{\eta}$ and half-width $\Delta$ : $G(\eta)=(\Delta / \pi) /\left[(\eta-\bar{\eta})^{2}+\Delta^{2}\right]$. In this case a maximal dimensionality reduction is achieved, since the integrals in Eqs. (6) and (10) can be evaluated applying the residue theorem. We analytically extend $w(\eta, t)$ into complex $\eta$ [32], close the integrals by an arc at infinity in the complex half-plane $\operatorname{Im}(\eta)<0$ [64], and obtain $\pi \tau r(t)+i v_{s}=$ $w(\bar{\eta}-i \Delta, t)$. Then, we evaluate Eq. (12) at $\eta=\bar{\eta}-i \Delta$, obtaining the FREs

$$
\begin{aligned}
\tau \dot{r} & =\frac{\Delta}{\tau \pi}+2 r v_{s}-g r, \\
\tau \dot{v}_{s} & =v_{s}^{2}+\bar{\eta}-(\pi \tau r)^{2}+(J+g \ln a) \tau r .
\end{aligned}
$$

This system of two ordinary differential equations for the mean firing rate $r$ and for the auxiliary variable $v_{s}$-see Eq. (11) - describes the dynamics of the ensemble exactly in the limit of infinite $N, V_{p}$, and $V_{r}$ The dual role of the electrical coupling $g$ is clearly described by the FREs: First, electrical coupling enters in Eq. (14a), reducing the firing rate, and hence reducing the width of the distribution of membrane potentials. This contribution homogenizes membrane potentials and, for large enough $g$, favors the emergence of collective synchronization [34]. Second, 


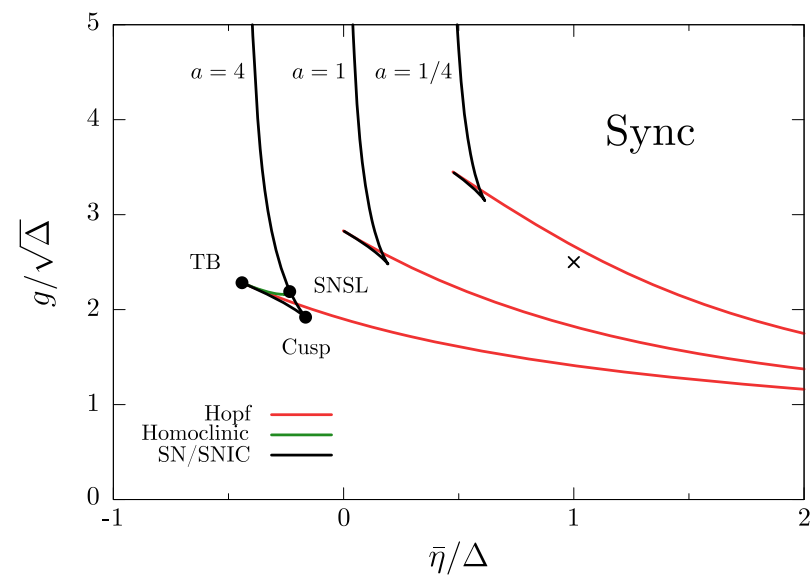

FIG. 2. Phase diagram of the FREs (14) for electrical coupling only $(J=0)$ and for three different values of the asymmetry parameter $a$. The region of synchronization ("sync"), located in the upper-right part of the diagram, enlarges as $a$ grows. Symbol $\times$ indicates the parameters value $(1,2.5)$, used in Fig. 3. To lighten the diagram, homoclinic bifurcations and codimension-2 pointscusp, saddle-node-separatrix-loop (SNSL), and Takens-Bogdanov (TB) - are omitted in the cases $a=1$ and $a=1 / 4$.

electrical coupling enters in Eq. (14b), contributing as a virtual chemical coupling. Depending on whether $a$ is larger or smaller than 1 , electrical and chemical synapses cooperate or compete for synchrony.

The relevant parameters of Eqs. (14) are $\bar{\eta}$, the coupling constants $J$ and $g$, and the spike asymmetry $a$ (one can assume $\Delta=1$ and $\tau=10 \mathrm{~ms}$ without loss of generality). Additionally, parameters $a$ and $J$ both act in the same term of Eq. (14) and hence they cannot produce qualitatively different dynamical behaviors. Accordingly, we consider $J=0$ hereafter and borrow the results of the bifurcation analysis in [34], which can be directly applied here replacing the chemical coupling constant $J$ by the effective chemical coupling Eq. (13) [65]. The phase diagram in Fig. 2 shows the bifurcation loci of the FREs for three values of $a$. Three bifurcations, Hopf (red), saddle-node (black), and homoclinic (green), tangentially meet at the Takens-Bogdanov (TB) point,

$$
(\bar{\eta} / \Delta)_{\mathrm{TB}}=-\frac{1}{\pi} \ln a,
$$

around which the phase diagram organizes. The homoclinic curve (shown only for $a=4$ ) moves parallel to the Hopf line for a while, and then tangentially meets the upper branch of the Saddle-Node (SN) bifurcating line at a saddle-node-separatrix-loop (SNSL) point. At this point, the SN boundary becomes a $\mathrm{SN}$ on the invariant circle (SNIC) boundary (black) that together with the Hopf and homoclinic lines encloses the region of synchronization (sync) where collective oscillations occur. Note that two small regions of bistability are located in the region limited by the three codimension-two points: cusp, TB, and SNSL.

In Fig. 2 the SNIC bifurcation lines have a vertical asymptote precisely at the same $\bar{\eta}$ value as the TB point, Eq. (15). For $0<a<1$, the asymptote is located at positive values of $\bar{\eta}$, and shifts to the left as $a$ increases. Hence, synchronization is hindered for spikes with $0<a<1$, and favored for spikes with $a>1$ [66]. In Fig. 3 we depict the time series of the firing rate in numerical simulations of both the network Eqs. (3) and the FREs Eqs. (14), for three values of the parameter $a=\{1 / 4,1,4\}$. In the first row, we used spikes with $V_{p}=100$ in Eqs. (3). Though the agreement between the models is not perfect, the mean-field Eqs. (14) accurately predict the emergence of oscillations in Eqs. (3) as $a$ is increased. Finally, for $V_{p}=1000$ (second row), the agreement greatly improves showing that the dynamics of the network Eqs. (3) converges to that of the FREs for large values of $V_{p}$.
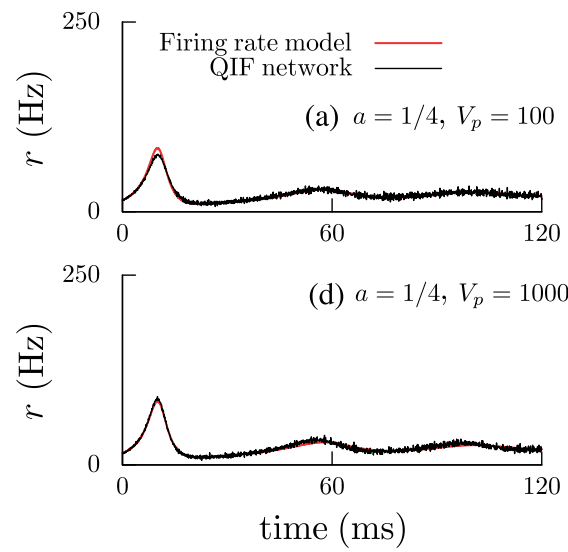
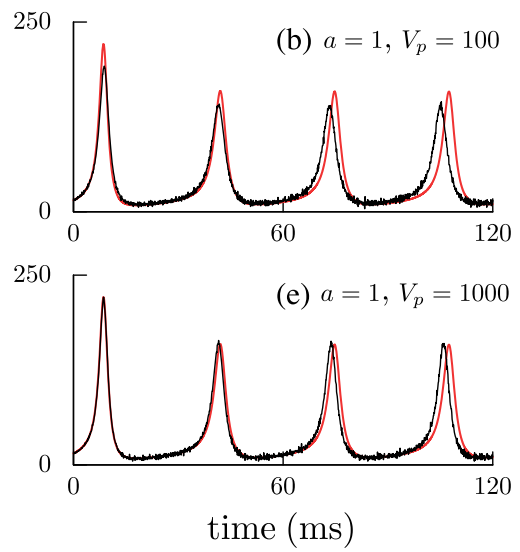
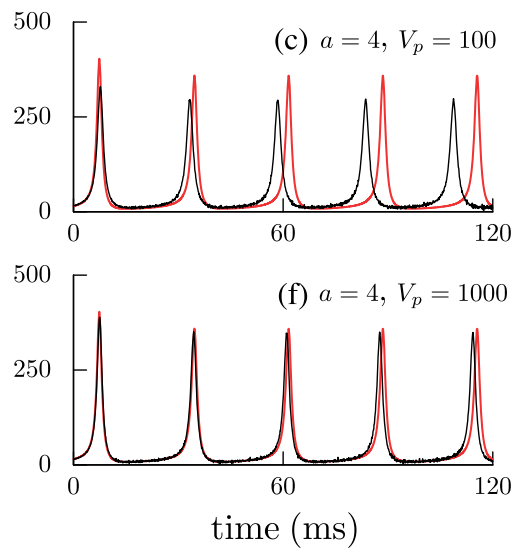

FIG. 3. Time series of $r(t)$ for FREs (14) (red) and network Eqs. (3) with $N=10^{4}$ (black). Peak values are (a)-(c) $V_{p}=100$, and (d)-(f) $V_{p}=1000$. Three values of the spike asymmetry parameter are used: (a),(d) $a=1 / 4$, (b), (e) $a=1$, (c),(f) $a=4$. Parameters correspond to the symbol $\times$ in Fig. $2: J=0, g=2.5, \bar{\eta}=\Delta=1$, and $\tau=10 \mathrm{~ms}$. We used the explicit Euler scheme with $d t=10^{-4} \mathrm{~ms}$ and $\tau_{s}=10^{-3} \mathrm{~ms}$. 
Conclusions.-We showed that the spike resetting rule of the QIF model can be incorporated in the mean-field theory proposed in [32]. This extension reveals a nontrivial dependence of the mean membrane voltage on the firing rate, Eq. (11), which turns out to be crucial for deciphering the role of electrical synapses in synchrony, and to reconcile previous theoretical and numerical work. Previous works have sought to model electrical coupling in integrate-and-fire models introducing, heuristically, an additional impulsive coupling contribution [17-22]. Remarkably, such contribution arises from first principles in our mean-field theory, and is the consequence of the asymmetry of the spikes. Moreover, as the FREs (14) are valid for arbitrary coupling strengths, we conclude that electrical and chemical coupling do not simply add linearly for weak coupling, as suggested in previous work, see, e.g., [23]. Finally, given that traditional mean-field theories only account for chemically coupled ensembles, we regard Eqs. (14) as a unique tool to investigate neural networks with both chemical and electrical synapses.

We acknowledge support by the Agencia Estatal de Investigación and Fondo Europeo de Desarrollo Regional under Projects No. PID2019-109918GB-I00 and No. FIS2016-74957-P (AEI/FEDER, EU).

[1] M. Galarreta and S. Hestrin, Nat. Rev. Neurosci. 2, 425 (2001).

[2] M. V. Bennett and R. Zukin, Neuron 41, 495 (2004).

[3] B. W. Connors and M. A. Long, Annu. Rev. Neurosci. 27, 393 (2004).

[4] J. I. Nagy, A. E. Pereda, and J. E. Rash, Biochim. Biophys. Acta, Bioenerg. 1860, 102 (2018).

[5] R. D. Traub, M. A. Whittington, R. Gutiérrez, and A. Draguhn, J. Cell Tissue Res. 373, 671 (2018).

[6] A. Draguhn, R. Traub, D. Schmitz, and J. Jefferys, Nature (London) 394, 189 (1998).

[7] P. Mann-Metzer and Y. Yarom, J. Neurosci. 19, 3298 (1999).

[8] J. L. Perez Velazquez and P. L. Carlen, Trends Neurosci. 23, 68 (2000).

[9] G. Tamás, E. H. Buhl, A. Lörincz, and P. Somogyi, Nat. Neurosci. 3, 366 (2000).

[10] M. R. Deans, J. R. Gibson, C. Sellitto, B. W. Connors, and D. L. Paul, Neuron 31, 477 (2001).

[11] S. G. Hormuzdi, I. Pais, F. E. LeBeau, S. K. Towers, A. Rozov, E. H. Buhl, M. A. Whittington, and H. Monyer, Neuron 31, 487 (2001).

[12] S. Stagkourakis, C. T. Pérez, A. Hellysaz, R. Ammari, and C. Broberger, eLife 7, e33144 (2018).

[13] C. Bou-Flores and A. J. Berger, J. Neurophysiol. 85, 1543 (2001).

[14] K. Vervaeke, A. Lrincz, P. Gleeson, M. Farinella, Z. Nusser, and R. A. Silver, Neuron 67, 435 (2010).

[15] B. W. Connors, Dev. Neurobiol. 77, 610 (2017).

[16] C. C. Chow and N. Kopell, Neural Comput. 12, 1643 (2000).
[17] T. J. Lewis and J. Rinzel, J. Comput. Neurosci. 14, 283 (2003).

[18] B. Pfeuty, G. Mato, D. Golomb, and D. Hansel, Neural Comput. 17, 633 (2005).

[19] B. Pfeuty, G. Mato, D. Golomb, and D. Hansel, J. Neurosci. 23, 6280 (2003).

[20] T. J. Lewis and F. K. Skinner, Understanding activity in electrically coupled networks using PRCs and the theory of weakly coupled oscillators, in Phase Response Curves in Neuroscience: Theory, Experiment, and Analysis, edited by N. W. Schultheiss, A. A. Prinz, and R. J. Butera (Springer, New York, NY, 2012), pp. 329-359.

[21] C. Börgers, An Introduction to Modeling Neuronal Dynamics (Springer, New York, 2017), Vol. 66.

[22] S. Ostojic, N. Brunel, and V. Hakim, J. Comput. Neurosci. 26, 369 (2009).

[23] N. Kopell and B. Ermentrout, Proc. Natl. Acad. Sci. U.S.A. 101, 15482 (2004).

[24] B. Pfeuty, D. Golomb, G. Mato, and D. Hansel, Front. Comput. Neurosci. 1, 8 (2007).

[25] X.-J. Wang and G. Buzsáki, J. Neurosci. 16, 6402 (1996).

[26] J. A. White, C. C. Chow, J. Rit, C. Soto-Treviño, and N. Kopell, J. Comput. Neurosci. 5, 5 (1998).

[27] P. Tiesinga and J. V. José, Network Comput. Neural Systems 11, 1 (2000).

[28] F. Devalle, A. Roxin, and E. Montbrió, PLoS Comput. Biol. 13, e1005881 (2017).

[29] H. R. Wilson and J. D. Cowan, Biophys. J. 12, 1 (1972).

[30] E. Ott and T. M. Antonsen, Chaos 18, 037113 (2008).

[31] T. B. Luke, E. Barreto, and P. So, Neural Comput. 25, 3207 (2013).

[32] E. Montbrió, D. Pazó, and A. Roxin, Phys. Rev. X 5, 021028 (2015).

[33] C. R. Laing, SIAM J. Appl. Dyn. Syst. 14, 1899 (2015).

[34] B. Pietras, F. Devalle, A. Roxin, A. Daffertshofer, and E. Montbrió, Phys. Rev. E 100, 042412 (2019).

[35] A. Daffertshofer and B. Pietras, Phase synchronization in neural systems, in Encyclopedia of Complexity and Systems Science, edited by R. A. Meyers (Springer Berlin Heidelberg, Berlin, Heidelberg, 2020), pp. 1-14.

[36] Á. Byrne, J. Ross, R. Nicks, and S. Coombes, bioRxiv (2020), https://doi.org/10.1101/2020.08.12.246256.

[37] P. So, T. B. Luke, and E. Barreto, Physica (Amsterdam) 267D, 16 (2014).

[38] C. R. Laing, Phys. Rev. E 90, 010901(R) (2014).

[39] J. Roulet and G. B. Mindlin, Chaos 26, 093104 (2016).

[40] D. Pazó and E. Montbrió, Phys. Rev. Lett. 116, 238101 (2016).

[41] I. Ratas and K. Pyragas, Phys. Rev. E 94, 032215 (2016).

[42] J. M. Esnaola-Acebes, A. Roxin, D. Avitabile, and E. Montbrió, Phys. Rev. E 96, 052407 (2017).

[43] G. Dumont, G. B. Ermentrout, and B. Gutkin, Phys. Rev. E 96, 042311 (2017).

[44] F. Devalle, E. Montbrió, and D. Pazó, Phys. Rev. E 98, 042214 (2018).

[45] H. Schmidt, D. Avitabile, E. Montbrió, and A. Roxin, PLoS Comput. Biol. 14, 1 (2018).

[46] M. di Volo and A. Torcini, Phys. Rev. Lett. 121, 128301 (2018).

[47] I. Ratas and K. Pyragas, Phys. Rev. E 98, 052224 (2018). 
[48] S. Coombes and Á. Byrne, in Nonlinear Dynamics in Computational Neuroscience, edited by F. Corinto and A. Torcini (Springer International Publishing, Cham, 2019), pp. $1-16$.

[49] A. Byrne, D. Avitabile, and S. Coombes, Phys. Rev. E 99, 012313 (2019).

[50] G. Dumont and B. Gutkin, PLoS Comput. Biol. 15, e1007019 (2019).

[51] S. Keeley, Á. Byrne, A. Fenton, and J. Rinzel, J. Neurophysiol. 121, 2181 (2019).

[52] H. Schmidt and D. Avitabile, Chaos 30, 033133 (2020).

[53] R. Gast, H. Schmidt, and T. R. Knoesche, Neural Comput. 32, 1615 (2020).

[54] M. Segneri, H. Bi, S. Olmi, and A. Torcini, Front. Comput. Neurosci. 14, 47 (2020).

[55] A. Ceni, S. Olmi, A. Torcini, and D. Angulo-Garcia, Chaos 30, 053121 (2020).

[56] H. Bi, M. Segneri, M. di Volo, and A. Torcini, Phys. Rev. Research 2, 013042 (2020).

[57] C. Bick, M. Goodfellow, C. R. Laing, and E. A. Martens, J. Math. Neurosci. 10, 9 (2020).

[58] E. M. Izhikevich, Dynamical Systems in Neuroscience (The MIT Press, Cambridge, Massachusetts, 2007).

[59] P. Latham, B. Richmond, P. Nelson, and S. Nirenberg, J. Neurophysiol. 83, 808 (2000).

[60] D. Hansel and G. Mato, Neural Comput. 15, 1 (2003).

[61] In Refs. [59] and [23], for example, the QIF model is analyzed using biophysically realistic values of $V_{p}$ and $V_{r}$.
The corresponding spike asymmetry ratios are $a \approx 3.4$ in [59], and $a \approx 15.2$ in [23]. Though action potentials satisfy $a>1$, here we also consider spikes with $a \leq 1$ for illustration purposes.

[62] B. Ermentrout and N. Kopell, SIAM J. Appl. Math. 46, 233 (1986).

[63] The integration limits cannot be simply replaced by $\pm \infty$, due to the slow decay of the Lorentzian distribution $\rho(V \mid \eta, t) V \sim V^{-1}$ as $|V| \rightarrow \infty$.

[64] The integrals along the arc at infinity are zero, since according to Eq. (12) we have $|w| \sim \sqrt{|\eta|}$. Accordingly, e.g., for Eq. (6), we have $\int_{0}^{-\pi} \operatorname{Re}(w) G(\eta)|\eta| i e^{i \phi} d \phi \sim|\eta|^{3 / 2} /|\eta|^{2} \rightarrow 0$; likewise for Eq. (10).

[65] The weakly nonlinear analysis performed in [34] also applies here, and confirms that - in contrast to [22]-the Hopf bifurcation remains always supercritical for any value of $a$.

[66] The asymptote of the SNIC boundary is located at $\bar{\eta}<0$ for $a>1$. Therefore, even if a majority of the neurons are intrinsically quiescent, electrical coupling is able to trigger a self-sustained collective oscillation. Similar effects have been reported for pairs of electrically coupled neurons [67,68].

[67] Y. Manor, J. Rinzel, I. Segev, and Y. Yarom, J. Neurophysiol. 77, 2736 (1997).

[68] T. Kepler, E. Marder, and L. Abbott, Science 248, 83 (1990). 